\title{
Real-World Injection Frequency and Cost of Ranibizumab and Aflibercept for the Treatment of Neovascular Age-Related Macular Degeneration and Diabetic Macular Edema
}

\author{
Szilárd Kiss, MD; Elisabetta Malangone-Monaco, MS; Kathleen Wilson, MPH; Helen Varker, BS; \\ Diana Stetsovsky, MS; David Smith, PhD; and Vincent Garmo, MHS
}

\begin{abstract}
BACKGROUND: Ranibizumab and aflibercept are FDA-approved treatments for patients with neovascular age-related macular degeneration (nAMD) and diabetic macular edema (DME). Although these agents differ in cost and labeled dosing, it is unclear whether these differences are reflected in clinical practice.
\end{abstract}

OBJECTIVE: To compare the real-world frequency and cost of ranibizumab and aflibercept injections among treatment-naive and previously treated patients with nAMD and DME.

METHODS: Claims data from MarketScan Research Databases were retrospectively reviewed to identify treatment-naive patients with nAMD who initiated intravitreal ranibizumab or aflibercept between January 1, 2014, and January 1, 2016, and treatment-naive patients with DME who initiated intravitreal ranibizumab or aflibercept between July 29, 2014, and July 1, 2016. Patients who switched to subsequent-line aflibercept or ranibizumab during the study period were eligible to enter previously treated subgroups. Multivariable regression models were derived to compare the per-patient frequency and cost of injections between ranibizumab- and aflibercepttreated patients with nAMD over 12 months (treatment-naive: $n=1,087$ and $n=1,578$; previously treated: $n=221$ and $n=751$ ) and 24 months (treatment-naive: $n=454$ and $n=568$; previously treated: $n=93$ and $n=284$ ) and in patients with DME over 6 months (treatment-naive: $n=507$ and $n=681$; previously treated: $n=53$ and $n=223$ ) and 12 months (treatment-naive: $n=326$ and $n=382$; previously treated: $n=24$ and $n=122$ ).

RESULTS: After adjusting for patient demographics and clinical characteristics, per-patient injection frequency and cost were not significantly different between treatment-naive patients with nAMD who received ranibizumab versus aflibercept over 12 months (5.62 vs. $5.54 ; P=0.52$, and $\$ 11,351$ vs. $\$ 10,702 ; P=0.06$, respectively) and 24 months (7.86 vs. 8.37; $P=0.16$, and $\$ 16,286$ vs. $\$ 16,666 ; P=0.69$, respectively). In previously treated patients with nAMD, injection frequency was significantly lower among ranibizumab- versus aflibercept-treated patients over 24 months (7.98 vs. 9.63; $P=0.03)$, whereas treatment costs were comparable over 12 months $(\$ 11,512$ vs. $\$ 12,050 ; P=0.44)$ and 24 months $(\$ 16,303$ vs. $\$ 19,361 ; P=0.13)$. In treatment-naive patients with DME, ranibizumab was associated with significantly fewer injections and lower costs than aflibercept over 6 months ( 2.60 vs. 2.92 and $\$ 3,379$ vs. $\$ 5,925$, respectively; both $P<0.001)$ and 12 months ( 3.33 vs. 3.87 and $\$ 4,136$ vs. $\$ 7,656$, respectively; both $P<0.001)$. Similar cost savings were observed among previously treated patients with DME who received ranibizumab over 6 months ( $\$ 3,834$ vs. $\$ 6,775$ for aflibercept; $P=0.0001)$ and 12 months $(\$ 4,606$ vs. $\$ 9,190 ; P=0.02$ ), despite nonsignificant differences in injection frequency during follow-up.

CONCLUSIONS: Although the frequency and cost of ranibizumab and aflibercept injections were generally comparable among patients treated for nAMD, ranibizumab was associated with estimated per-patient-per-year cost savings of $\$ 3,500-\$ 4,500$ in those treated for DME. Most patients received fewer injections than any FDA-indicated dosing schedule, suggesting potential undertreatment that may result in suboptimal vision outcomes.

J Manag Care Spec Pharm. 2020;26(3):253-66

Copyright $\odot 2020$, Academy of Managed Care Pharmacy. All rights reserved.

\section{What is already known about this subject}

- Aflibercept $2.0 \mathrm{mg}$ (wholesale acquisition cost [WAC] \$1,850) is approved for the treatment of neovascular age-related macular degeneration (nAMD) and diabetic macular edema (DME), whereas ranibizumab $0.5 \mathrm{mg}$ (WAC \$1,950) and $0.3 \mathrm{mg}$ (WAC $\$ 1,170$ ) are indicated for $\mathrm{nAMD}$ and DME, respectively.

Prescribing information for ranibizumab and aflibercept in nAMD and DME suggest that, over time, comparatively fewer aflibercept injections are required to achieve optimal patient outcomes.

Evidence suggests that retina specialists are more likely to administer treatment according to "treat and observe" or "treat and extend" protocols; as such, real-world injection frequencies are similar between ranibizumab and aflibercept and lower than labeled dosing schedules.

\section{What this study adds}

This study evaluates real-world patterns of ranibizumab and aflibercept therapy in treatment-naive and previously treated patients with nAMD and DME.

Compared with aflibercept, ranibizumab was estimated to be cost saving in treatment-naive and previously treated patients with DME over 6 and 12 months; savings were driven by the significantly lower cost of ranibizumab $0.3 \mathrm{mg}$ versus aflibercept $2.0 \mathrm{mg}$.

Data suggest that a significant proportion of patients in clinical practice receive fewer than the recommended number of ranibizumab and aflibercept injections for nAMD and DME, highlighting potential undertreatment that may lead to suboptimal vision outcomes. 


\section{Real-World Injection Frequency and Cost of Ranibizumab and Aflibercept for the Treatment of Neovascular Age-Related Macular Degeneration and Diabetic Macular Edema}

A ge-related macular degeneration and diabetic retinopathy are leading causes of blindness in the United States. ${ }^{1-3}$ The burden associated with these conditions is largely attributed to progressive disease and development of neovascular age-related macular degeneration (nAMD) and diabetic macular edema (DME), respectively. In nAMD, exudative choroidal neovascularization leads to irreversible central vision loss, ${ }^{4}$ whereas DME is characterized by swelling or thickening of the macula and subsequent central vision impairment. ${ }^{5}$ Vascular endothelial growth factor (VEGF) is implicated in nAMD and DME pathogeneses due to its key role in regulating angiogenesis and vascular permeability. ${ }^{4,5}$ Increased VEGF expression mediates choroidal neovascularization characteristic of nAMD, ${ }^{4,6-8}$ leading to exudate leakage, hemorrhage, and subretinal scarring. In DME, VEGF overexpression promotes increased retinal vascular permeability, ${ }^{5,9,10}$ resulting in fluid and protein accumulation in the macula. Intravitreal antiVEGF therapy has been shown to improve visual and anatomic outcomes in patients with nAMD and DME and has subsequently become the standard of care for these conditions. ${ }^{11,12}$

At present, 2 anti-VEGF agents are approved by the U.S. Food and Drug Administration (FDA) for the treatment of nAMD and DME. Ranibizumab was approved for nAMD in 2006 and DME in 2012, ${ }^{13}$ whereas aflibercept was approved for nAMD in 2011 and DME in 2014. ${ }^{14}$ Other anti-VEGF therapies include pegaptanib, approved for nAMD in 2004 and used off-label for DME ${ }^{15}$ and bevacizumab, used off-label for both nAMD and DME. ${ }^{16}$ Intravitreal injections of ranibizumab and aflibercept provide similar therapeutic benefits in patients with nAMD ${ }^{17,18}$ and $\mathrm{DME}^{19,20}$; however, they differ in wholesale acquisition cost (WAC) and FDA-approved dosage and administration.

Approved labeling for ranibizumab and aflibercept each describe 3 injection schedules for nAMD. In this indication, ranibizumab $0.5 \mathrm{mg}$ (WAC $\$ 1,950$ ) is recommended monthly ( $44 \mathrm{w} ; \approx 12$ injections over 1 year). Alternatively, patients may receive ranibizumab $0.5 \mathrm{mg}$ as needed (pro re nata [PRN]) after 3 monthly loading doses or 1 dose every 3 months (q12w) after 4 monthly loading doses; however, these regimens may be less effective. ${ }^{13}$ In comparison, aflibercept $2.0 \mathrm{mg}$ (WAC \$1,850) is recommended once every 2 months (q8w) after 3 monthly loading doses ( $\approx 8$ injections in the first year). More frequent dosing of aflibercept $2.0 \mathrm{mg}(\mathrm{q} 4 \mathrm{w})$ is approved but provides no additional efficacy, whereas less frequent dosing (q12w after 12 months of effective therapy) is approved but may be less effective. ${ }^{14}$

In DME, ranibizumab $0.3 \mathrm{mg}$ (WAC $\$ 1,170$ ) is recommended q4w ( $\approx 12$ injections over 1 year), ${ }^{13}$ whereas aflibercept $2.0 \mathrm{mg}$ is recommended $\mathrm{q} 8 \mathrm{w}$ after 5 monthly loading doses $(\approx 9$ injections in the first year). Although aflibercept $2.0 \mathrm{mg}$ may be administered more frequently ( $q 4 \mathrm{w}$ ), no additional efficacy has been demonstrated with this regimen. ${ }^{14}$
Differences in cost and injection frequency between ranibizumab and aflibercept likely account for differences in health care resource utilization and expenditure. However, it is unclear whether treatment patterns in routine clinical practice reflect FDA-approved labeling. Preferences and Trends Surveys conducted by the American Society of Retina Specialists indicate that physicians are more likely to treat nAMD based on optical coherence tomography measures of disease activity rather than vision loss or labeled treatment schedules. ${ }^{21,22}$ For example, 2 common treatment protocols for nAMD include "treat and observe," where injections are administered PRN upon signs of recurrent exudation, and "treat and extend," where injection intervals are extended while the macula remains dry. ${ }^{22,23}$ Although PRN treatment of nAMD is approved for ranibizumab, treat and extend regimens are not recommended for either ranibizumab or aflibercept. ${ }^{13,14}$

Recent studies of treatment-naive patients with nAMD found that the frequency and cost of aflibercept and ranibizumab injections were comparable in the first year of treatment $^{24,25}$; however, there is a paucity of data over longer time periods, in previously treated patients, and in the DME population. This retrospective analysis of U.S. claims data compared the real-world frequency and cost of ranibizumab and aflibercept injections among treatment-naive and previously treated patients with nAMD and DME.

\section{Methods}

\section{Data Source}

Health insurance claims data from January 1, 2013, to December 31, 2016 (study period), were extracted from the IBM MarketScan Commercial Claims and Encounters (Commercial), Medicare Supplemental and Coordination of Benefits (Medicare Supplemental), and Early View databases (IBM Watson Health, Cambridge, MA). These databases contain information relating to adjudicated inpatient, outpatient, and drug prescription claims for the U.S. working population, their dependents, and retirees with employer-sponsored primary or Medicare supplemental insurance. Data stored within MarketScan Research Databases are statistically deidentified and Health Insurance Portability and Accountability Act compliant. ${ }^{26}$

\section{Study Population}

Patients in the nAMD cohort initiated intravitreal ranibizumab or aflibercept between January 1, 2014, and January 1, 2016 (allowing $\geq 12$ months of follow-up). This period was intended to capture recent treatment patterns in an adequate number of patients with $\geq 24$ months of follow-up. Patients in the DME cohort initiated intravitreal ranibizumab or aflibercept between July 29, 2014 (FDA approval date for aflibercept in DME), and July 1, 2016 (allowing $\geq 6$ months of follow-up) Index dates for each patient were defined as the date of first 
exposure to ranibizumab or aflibercept, and the 12 months preceding each index date was designated the baseline period.

Included patients were aged $\geq 18$ years at the index date and had continuous medical and prescription insurance throughout the baseline period. Patients with no evidence of bevacizumab, ranibizumab, aflibercept, or pegaptanib treatment between January 1, 2005, and the index date were initially included in treatment-naive subgroups and were subsequently eligible for inclusion in previously treated subgroups after switching antiVEGF therapy during the study period. Patients with nAMD or DME were identified by International Classification of Diseases, Ninth/Tenth Revision, Clinical Modification (ICD-9/10-CM) diagnosis codes recorded at the index date or during the baseline period; evidence of intravitreal anti-VEGF treatment was based on Healthcare Common Procedure Coding System (HCPCS) and Current Procedural Terminology codes (available from the authors upon request)..$^{27,28}$

Patients with outpatient prescription claims for ranibizumab, bevacizumab, aflibercept, or pegaptanib treatment at any point in their administrative claims history and/or evidence of bilateral intravitreal anti-VEGF treatment during the anti-VEGF exposure period were excluded. Patients with ICD-9/10-CM codes for DME or retinal vein occlusion during baseline or follow-up periods were excluded from the nAMD cohort; those with diagnosis codes for nAMD or retinal vein occlusion were excluded from the DME cohort.

\section{Follow-Up and Outcome Measures}

Follow-up was defined as the time from the index date until treatment was switched to a different anti-VEGF agent, disenrollment from health insurance, inpatient death, or study end, whichever occurred first. A study end date of December 31 , 2016, ensured that $\geq 95 \%$ of claims during follow-up were adjudicated at the time of data extraction. Treatmentnaive patients who switched to subsequent-line aflibercept or ranibizumab during the study period were eligible to enter previously treated subgroups (with associated index date and baseline periods), provided their follow-up period was of sufficient duration.

Analyses compared ranibizumab and aflibercept therapy across 8 different subgroups: treatment-naive and previously treated nAMD over 12 and 24 months and treatment-naive and previously treated DME over 6 and 12 months. Primary outcomes were per-patient frequency and cost of ranibizumab and aflibercept injections in each subgroup. Treatment costs were measured using the payment fields on extracted claims and included gross covered payments for the anti-VEGF agent alone (i.e., amount eligible for payment after applying pricing guidelines such as fee schedules and discounts). Costs associated with intravitreal administration were not considered because they were assumed to be identical for ranibizumab and aflibercept.
In secondary analyses, the number of ranibizumab and aflibercept injections received by treatment-naive patients were grouped according to FDA-indicated injection schedules. For treatment-naive patients with nAMD, the number of injections received over 12 months was categorized as follows: (a) $\geq 11$ injections, to reflect ranibizumab and aflibercept $\mathrm{q} 4 \mathrm{w}$ regimens $(\approx 12 \text { injections })^{13,14}$; (b) $\geq 7$ injections, reflecting PRN ranibizumab in HARBOR (mean 7.7 injections), ${ }^{29}$ ranibizumab q12w after 4 monthly loading doses ( $\approx 7$ injections), ${ }^{13}$ and aflibercept q8w after 3 monthly loading doses $(\approx 8 \text { injections })^{14}$; (c) $\geq 3$ injections, to reflect loading doses in ranibizumab $\mathrm{q} 12 \mathrm{w}$ and aflibercept q8w regimens; or (d) 1-2 injections, to capture other dosing scenarios. For treatment-naive patients with nAMD and 24 months of follow-up, injection frequency was grouped as follows: (a) $\geq 23$ injections, to reflect ranibizumab and aflibercept $\mathrm{q} 4 \mathrm{w}$ regimens $(\approx 24 \text { injections })^{13,14}$; (b) $\geq 13$ injections, reflecting PRN ranibizumab in HARBOR (mean 13.3 injections) ${ }^{30}$ and aflibercept $\mathrm{q} 8 \mathrm{w}$ after 3 monthly loading doses ( $\approx 14$ injections $)^{14}$; (c) $\geq 3$ injections, to reflect loading doses in PRN ranibizumab and aflibercept q8w regimens; or (d) 1-2 injections, to capture other dosing scenarios.

For treatment-naive patients with DME, the number of ranibizumab and aflibercept injections received over 12 months was categorized as follows: (a) $\geq 11$ injections, to reflect ranibizumab and aflibercept $\mathrm{q} 4 \mathrm{w}$ regimens $(\approx 12$ injections $)^{13,14}$; (b) $\geq 8$ injections, reflecting aflibercept $\mathrm{q} 8 \mathrm{w}$ after 5 monthly loading doses $(\approx 9 \text { injections })^{14}$; (c) $\geq 5$ injections, reflecting aflibercept q8w loading doses; or (d) 1-4 injections, to capture other dosing scenarios. Injection thresholds for each dosing category included an allowable margin of 1 injection to account for strict cutoffs to follow-up in this study (e.g., q4w dosing over 12 months was defined as $\geq 11$ injections, rather than $\geq 12$ injections).

\section{Covariates}

Patient demographics measured at the index date included age, sex, U.S. Census Bureau geographic region, urban or rural residence, insurance plan type, primary payer, and median household income. Clinical characteristics measured throughout the baseline period included nonophthalmic comorbidities and medications (nonmelanoma cancer, hypertension, diabetes, dyslipidemia, nonsteroidal anti-inflammatory drugs, and glucocorticoids); ophthalmic comorbidities and treatments (cataract, glaucoma, cataract surgery, and intravitreal steroid injections); and general health status indices (Deyo Charlson Comorbidity Index, ${ }^{31}$ unique ICD-9/10-CM diagnosis codes, unique National Drug Code numbers, ${ }^{32}$ and total health care expenditure).

\section{Statistical Analyses}

For each patient subgroup, data were reported using bivariate descriptive summary statistics, stratified by treatment 


\section{Real-World Injection Frequency and Cost of Ranibizumab and Aflibercept for the Treatment of Neovascular Age-Related Macular Degeneration and Diabetic Macular Edema}

\section{TABLE 1 Baseline Demographics and Clinical Measurements of RBZ- and AFL-Treated}

Patients with nAMD

\begin{tabular}{|c|c|c|c|c|c|c|c|c|}
\hline \multirow[b]{3}{*}{ Characteristic } & \multicolumn{4}{|c|}{ Treatment-Naive nAMD } & \multicolumn{4}{|c|}{ Previously Treated nAMD } \\
\hline & \multicolumn{2}{|c|}{$\geq 12$ Months of Follow-up } & \multicolumn{2}{|c|}{$\geq 24$ Months of Follow-up } & \multicolumn{2}{|c|}{$\geq 12$ Months of Follow-up } & \multicolumn{2}{|c|}{$\geq 24$ Months of Follow-up } \\
\hline & $\operatorname{RBZ}(\mathrm{n}=1,087)$ & $\operatorname{AFL}(\mathrm{n}=1,578)$ & $\mathrm{RBZ}(\mathrm{n}=454)$ & $\operatorname{AFL}(\mathrm{n}=568)$ & $\operatorname{RBZ}(\mathrm{n}=221)$ & $\operatorname{AFL}(n=751)$ & RBZ (n=93) & $\operatorname{AFL}(n=284)$ \\
\hline \multicolumn{9}{|l|}{ Patient demographics ${ }^{a}$} \\
\hline Age, years, mean (SD) & $79.8(10.4)$ & $79.2(10.1)$ & $79.8 \quad(9.8)$ & $79.5(10.0)$ & $78.1(10.6)$ & $78.0(10.4)$ & $76.5(12.0)$ & $77.5(10.7)$ \\
\hline Female, n (\%) & $685(63.0)$ & $985(62.4)$ & $296(65.2)$ & $364(64.1)$ & $135(61.1)$ & $425(56.6)$ & $56(60.2)$ & $152(53.5)$ \\
\hline \multicolumn{9}{|l|}{ Index year, n (\%) } \\
\hline 2014 & $608(55.9)$ & $677(42.9)$ & $454 \quad(100)$ & $564(99.3)$ & $129(58.4)$ & $365(48.6)$ & $93 \quad(100)$ & $283(99.6)$ \\
\hline 2015 & $479(44.1)$ & $901 \quad(57.1)$ & $(0)$ & $4 \quad(0.7)$ & $92(41.6)$ & $386(51.4)$ & $(0)$ & $1 \quad(0.4)$ \\
\hline \multicolumn{9}{|l|}{ Geographic region, n (\%) } \\
\hline Northeast & $280(25.8)$ & $470(29.8)$ & $124(27.3)$ & $151(26.6)$ & $64(29.0)$ & $235(31.3)$ & $25(26.9)$ & $97(34.2)$ \\
\hline Midwest & $377(34.7)$ & $588(37.3)$ & $167(36.8)$ & $232(40.8)$ & $52(23.5)$ & $216(28.8)$ & $23(24.7)$ & $85(29.9)$ \\
\hline South & $329(30.3)$ & $432(27.4)$ & $130(28.6)$ & $155(27.3)$ & $74(33.5)$ & $222(29.6)$ & $31 \quad(33.3)$ & $74(26.1)$ \\
\hline West & $97 \quad(8.9)$ & $87 \quad(5.5)$ & $31 \quad(6.8)$ & $29 \quad(5.1)$ & $31(14.0)$ & $78(10.4)$ & $14(15.1)$ & $28 \quad(9.9)$ \\
\hline Unknown & $4 \quad(0.4)$ & $1 \quad(0.1)$ & $2 \quad(0.4)$ & $1 \quad(0.2)$ & $0 \quad(0)$ & $(0)$ & $(0)$ & $(0)$ \\
\hline \multicolumn{9}{|l|}{ Population density, n (\%) } \\
\hline Urban & $940(86.5)$ & $1,346(85.3)$ & $396(87.2)$ & $476(83.8)$ & $193(87.3)$ & $633(84.3)$ & $78(83.9)$ & $237(83.5)$ \\
\hline Rural & $143(13.2)$ & $232(14.7)$ & $56(12.3)$ & $92(16.2)$ & $28(12.7)$ & $118(15.7)$ & $15(16.1)$ & $47(16.5)$ \\
\hline Unknown & $4 \quad(0.4)$ & $0 \quad(0)$ & $2 \quad(0.4)$ & $0 \quad(0)$ & $0 \quad(0)$ & $0 \quad(0)$ & $0 \quad(0)$ & $0 \quad(0)$ \\
\hline \multicolumn{9}{|l|}{ Health plan type, n (\%) } \\
\hline FFS & $424(39.0)$ & $625(39.6)$ & $179(39.4)$ & $232(40.8)$ & $76 \quad(34.4)$ & $255(34.0)$ & $28(30.1)$ & $100(35.2)$ \\
\hline $\mathrm{HMO}$ & $43 \quad(4.0)$ & $47 \quad(3.0)$ & $20 \quad(4.4)$ & $17 \quad(3.0)$ & $21 \quad(9.5)$ & $61 \quad(8.1)$ & $10(10.8)$ & $18 \quad(6.3)$ \\
\hline POS & $54 \quad(5.0)$ & $67 \quad(4.2)$ & $28 \quad(6.2)$ & $19 \quad(3.3)$ & $9 \quad(4.1)$ & $37 \quad(4.9)$ & $2(2.2)$ & $9 \quad(3.2)$ \\
\hline $\mathrm{PPO}$ & $532(48.9)$ & $798(50.6)$ & $215 \quad(47.4)$ & $294(51.8)$ & $107(48.4)$ & $381(50.7)$ & $48(51.6)$ & $151(53.2)$ \\
\hline Other & $34 \quad(3.1)$ & $41 \quad(2.6)$ & $12 \quad(2.6)$ & $6 \quad(1.1)$ & $8 \quad(3.6)$ & $17 \quad(2.3)$ & $5 \quad(5.4)$ & $6 \quad(2.1)$ \\
\hline \multicolumn{9}{|l|}{ Primary payer type, n (\%) } \\
\hline Commercial & $112(10.3)$ & $154 \quad(9.8)$ & $41 \quad(9.0)$ & $47 \quad(8.3)$ & $30(13.6)$ & $98(13.0)$ & $17(18.3)$ & $40(14.1)$ \\
\hline Medicare & $975(89.7)$ & $1,424(90.2)$ & $413(91.0)$ & $521 \quad(91.7)$ & $191(86.4)$ & $653(87.0)$ & $76(81.7)$ & $244(85.9)$ \\
\hline $\begin{array}{l}\text { Median household } \\
\text { income, USD, mean (SD) }\end{array}$ & $\begin{array}{l}51,939 \\
(17,911) \\
\end{array}$ & $\begin{array}{l}49,131 \\
(17,114) \\
\end{array}$ & $\begin{array}{c}52,227 \\
(18,599) \\
\end{array}$ & $\begin{array}{c}48,655 \\
(16,649) \\
\end{array}$ & $\begin{array}{c}50,659 \\
(18,566) \\
\end{array}$ & $\begin{array}{l}49,754 \\
(17,442) \\
\end{array}$ & $\begin{array}{c}49,724 \\
(18,393) \\
\end{array}$ & $\begin{array}{l}49,216 \\
(17,209) \\
\end{array}$ \\
\hline \multicolumn{9}{|l|}{ Clinical measurements $^{\mathrm{b}}$} \\
\hline \multicolumn{9}{|c|}{ Nonophthalmic comorbidities and medications, $\mathrm{n}(\%)$} \\
\hline Nonmelanoma cancer & $124(11.4)$ & $174(11.0)$ & $55(12.1)$ & $61(10.7)$ & $27(12.2)$ & 87 (11.6) & $8 \quad(8.6)$ & $26 \quad(9.2)$ \\
\hline Dyslipidemia & $736(67.7)$ & $1,074(68.1)$ & $311(68.5)$ & $372(65.5)$ & $147(66.5)$ & $506 \quad(67.4)$ & $62(66.7)$ & $179(63.0)$ \\
\hline NSAIDs & $134(12.3)$ & $211(13.4)$ & $46(10.1)$ & $75(13.2)$ & $23(10.4)$ & 87 (11.6) & $14(15.1)$ & $24 \quad(8.5)$ \\
\hline Glucocorticoids & $129(11.9)$ & $212(13.4)$ & $51(11.2)$ & $74(13.0)$ & $34(15.4)$ & $101(13.4)$ & $15(16.1)$ & $32(11.3)$ \\
\hline Diabetes (type 1 or 2) & $202(18.6)$ & $324(20.5)$ & $92(20.3)$ & $112(19.7)$ & $34(15.4)$ & $161(21.4)$ & $16(17.2)$ & $62(21.8)$ \\
\hline \multicolumn{9}{|c|}{ Ophthalmic comorbidities and treatments, n (\%) } \\
\hline Cataracts & $403(37.1)$ & $626(39.7)$ & $168(37.0)$ & $201(35.4)$ & $79(35.7)$ & $269(35.8)$ & $36(38.7)$ & $104(36.6)$ \\
\hline Glaucoma & $249(22.9)$ & $340(21.5)$ & $111(24.4)$ & $123(21.7)$ & $47(21.3)$ & $156(20.8)$ & $19(20.4)$ & $55(19.4)$ \\
\hline Cataract surgery & $107 \quad(9.8)$ & $157 \quad(9.9)$ & $46(10.1)$ & $56 \quad(9.9)$ & $19 \quad(8.6)$ & $69 \quad(9.2)$ & $9 \quad(9.7)$ & $24 \quad(8.5)$ \\
\hline $\begin{array}{l}\text { Intravitreal steroid } \\
\text { injection }\end{array}$ & $7 \quad(0.6)$ & $9 \quad(0.6)$ & $4 \quad(0.9)$ & $4 \quad(0.7)$ & $5 \quad(2.3)$ & $9 \quad(1.2)$ & $2(2.2)$ & $3 \quad(1.1)$ \\
\hline
\end{tabular}

(aflibercept or ranibizumab). Poisson quasi-likelihood multivariable regression was used to model the frequency of ranibizumab and aflibercept injections during follow-up, and incidence rate ratios were estimated using ranibizumab as the reference category. To handle overdispersion in the outcome distribution, a scale parameter, estimated by the square root of deviance divided by degrees of freedom, was used to adjust the regression. Similarly, multivariable generalized linear models with a log link and gamma error distribution were used to model the cost of ranibizumab and aflibercept injections during follow-up, and cost ratios were estimated using ranibizumab as the reference category. The recycled prediction method was used to generate predicted mean cost differences between ranibizumab- and aflibercept-treated patients. Models 
Real-World Injection Frequency and Cost of Ranibizumab and Aflibercept for the Treatment

of Neovascular Age-Related Macular Degeneration and Diabetic Macular Edema

TABLE 1 Baseline Demographics and Clinical Measurements of RBZ- and AFL-Treated Patients with nAMD (continued)

\begin{tabular}{|c|c|c|c|c|c|c|c|c|}
\hline \multirow[b]{3}{*}{ Characteristic } & \multicolumn{4}{|c|}{ Treatment-Naive nAMD } & \multicolumn{4}{|c|}{ Previously Treated nAMD } \\
\hline & \multicolumn{2}{|c|}{$\geq 12$ Months of Follow-up } & \multicolumn{2}{|c|}{$\geq 24$ Months of Follow-up } & \multicolumn{2}{|c|}{$\geq 12$ Months of Follow-up } & \multicolumn{2}{|c|}{$\geq 24$ Months of Follow-up } \\
\hline & $\operatorname{RBZ}(\mathrm{n}=1,087)$ & $\operatorname{AFL}(n=1,578)$ & RBZ $(n=454)$ & AFL $(n=568)$ & $\operatorname{RBZ}(\mathrm{n}=221)$ & $\operatorname{AFL}(\mathbf{n}=751)$ & RBZ (n= 93) & AFL $(n=284)$ \\
\hline \multicolumn{9}{|c|}{ Health status indices, mean (SD) } \\
\hline $\begin{array}{l}\text { Deyo Charlson } \\
\text { Comorbidity Index }\end{array}$ & $1.5 \quad(1.9)$ & $1.4 \quad(1.7)$ & $1.5 \quad(1.9)$ & $1.3 \quad(1.7)$ & $1.4 \quad(1.9)$ & $1.4 \quad(1.6)$ & $1.1 \quad(1.5)$ & $1.4 \quad(1.6)$ \\
\hline $\begin{array}{l}\text { Unique ICD-9/10-CM } \\
\text { diagnoses }\end{array}$ & $18.2(11.0)$ & $17.8(10.3)$ & $16.9(10.3)$ & $16.1 \quad(9.0)$ & $17.1(10.1)$ & $17.3 \quad(9.9)$ & $15.7(10.8)$ & $16.2 \quad(9.5)$ \\
\hline $\begin{array}{l}\text { Unique National Drug } \\
\text { Code numbers }\end{array}$ & $12.3 \quad(8.6)$ & $11.9 \quad(8.1)$ & $12.4 \quad(8.9)$ & $11.7 \quad(7.7)$ & $11.4 \quad(7.9)$ & $11.2 \quad(7.7)$ & $11.8 \quad(8.8)$ & $10.6 \quad(7.3)$ \\
\hline $\begin{array}{l}\text { Total health care } \\
\text { expenditure, USD }\end{array}$ & $\begin{array}{c}21,125 \\
(47,866) \\
\end{array}$ & $\begin{array}{l}18,304 \\
(34,715)\end{array}$ & $\begin{array}{c}19,143 \\
(37,813)\end{array}$ & $\begin{array}{c}18,693 \\
(43,423)\end{array}$ & $\begin{array}{l}23,096 \\
(41,554)\end{array}$ & $\begin{array}{c}21,292 \\
(28,252)\end{array}$ & $\begin{array}{l}21,225 \\
(33,782) \\
\end{array}$ & $\begin{array}{c}21,031 \\
(31,439)\end{array}$ \\
\hline $\begin{array}{l}\text { Median total health } \\
\text { care expenditure, USD }\end{array}$ & 8,791 & 8,469 & 8,203 & 7,977 & 11,857 & 14,765 & 10,715 & 14,001 \\
\hline \multicolumn{9}{|c|}{ Unadjusted frequency and cost of anti-VEGF injections ${ }^{c}$} \\
\hline $\begin{array}{l}\text { Injection frequency, } \\
\text { mean (SD) }\end{array}$ & $5.60(3.11)$ & $5.56(2.95)$ & $7.88(5.69)$ & $8.35(5.53)$ & $5.95(3.24)$ & $6.09(3.06)$ & $8.04(6.27)$ & $9.60(5.98)$ \\
\hline$P$ value & \multicolumn{2}{|c|}{0.70} & \multicolumn{2}{|c|}{0.18} & \multicolumn{2}{|c|}{0.53} & \multicolumn{2}{|c|}{0.03} \\
\hline $\begin{array}{l}\text { Injection cost, USD, } \\
\text { mean (SD) }\end{array}$ & $\begin{array}{l}11,383 \\
(7,805)\end{array}$ & $\begin{array}{l}10,681 \\
(8,178)\end{array}$ & $\begin{array}{c}16,515 \\
(14,833)\end{array}$ & $\begin{array}{c}16,510 \\
(14,995)\end{array}$ & $\begin{array}{l}11,617 \\
(7,975)\end{array}$ & $\begin{array}{l}12,007 \\
(7,904)\end{array}$ & $\begin{array}{c}16,544 \\
(16,193)\end{array}$ & $\begin{array}{c}19,120 \\
(13,465)\end{array}$ \\
\hline$P$ value & \multicolumn{2}{|c|}{0.03} & \multicolumn{2}{|c|}{1.00} & \multicolumn{2}{|c|}{0.52} & \multicolumn{2}{|c|}{0.13} \\
\hline \multicolumn{9}{|c|}{$\begin{array}{l}\text { aPatient demographics were measured at the index date for each anti-VEGF treatment exposure. } \\
{ }^{b} \text { Clinical measurements were measured during the } 12 \text {-month baseline period for each anti-VEGF treatment exposure. } \\
\text { 'Unadjusted injection frequencies and costs were measured over the specified length of follow-up (12 and } 24 \text { months). In patients with }>12 \text { and }>24 \text { months of follow-up, } \\
\text { data were censored after the first } 12 \text { and } 24 \text { months, respectively. } \\
\text { AFL = aflibercept; FFS = fee-for-service; HMO=health maintenance organization; ICD-9/10-CM=International Classification of Diseases, Ninth/Tenth Revision, Clinical } \\
\text { Modification; } n A M D=\text { neovascular age-related macular degeneration; NSAID =nonsteroidal anti-inflammatory drug; POS=point-of-service; PPO=preferred provider } \\
\text { organization; RBZ= ranibizumab; SD=standard deviation; USD =U.S. dollars; VEGF=vascular endothelial growth factor. }\end{array}$} \\
\hline
\end{tabular}

were fitted separately for treatment-naive and previously treated subgroups. To avoid treatment bias and account for possible differences between patients likely to receive ranibizumab versus aflibercept, analyses were adjusted for patient demographics, baseline clinical characteristics, indices of general health status, baseline treatments, and other covariates. Statistical significance was defined as $P \leq 0.05$.

\section{Results}

\section{Study Population}

A total of 173,434 patients were eligible for inclusion in the nAMD cohort (Appendix A, available in online article). After exclusions, 24,200 patients who initiated anti-VEGF therapy were identified, of whom 6,262 (25.9\%) were treatment-naive and first received ranibizumab or aflibercept therapy on or after January 1, 2014. Excluding those with insufficient followup of $<12$ months, 1,087 ranibizumab-treated and 1,578 aflibercept-treated patients had $\geq 12$ months of follow-up; 454 ranibizumab-treated and 568 aflibercept-treated patients had $\geq 24$ months of follow-up.

Among the 24,200 patients with nAMD identified, 2,609 (10.8\%) switched to subsequent-line aflibercept or ranibizumab during the study period and were eligible to enter previously treated subgroups. Of these, 221 ranibizumab-treated and 751 aflibercept-treated patients were included in 12-month analyses, whereas 93 ranibizumab-treated and 284 aflibercept-treated patients were included in 24-month analyses. Across all nAMD subgroups, minor differences were observed for geographic region and index year among patients with $\geq 12$ months of follow-up; otherwise, baseline demographics and clinical measurements were well balanced between treatments (Table 1).

In total, 96,620 patients were eligible for inclusion in the DME cohort (Appendix B, available in online article). After exclusions, 6,156 patients who initiated anti-VEGF therapy were identified, of whom 2,100 (34.1\%) were treatment-naive and first received ranibizumab or aflibercept therapy on or after July 29, 2014. Excluding those with insufficient follow-up of $<6$ months, 507 ranibizumab-treated and 681 aflibercepttreated patients had $\geq 6$ months of follow-up; 326 ranibizumabtreated and 382 aflibercept-treated patients had $\geq 12$ months of follow-up.

Among the 6,156 patients with DME identified, 531 (8.6\%) switched to subsequent-line ranibizumab or aflibercept therapy during the study period and were eligible to enter previously treated subgroups. Of these, 53 ranibizumab-treated and 223 aflibercept-treated patients were included in 6-month analyses, 


\section{Real-World Injection Frequency and Cost of Ranibizumab and Aflibercept for the Treatment of Neovascular Age-Related Macular Degeneration and Diabetic Macular Edema}

\section{TABLE 2 Baseline Demographics and Clinical Measurements of RBZ- and AFL-Treated}

Patients with DME

\begin{tabular}{|c|c|c|c|c|c|c|c|c|}
\hline \multirow[b]{3}{*}{ Characteristic } & \multicolumn{4}{|c|}{ Treatment-Naive DME } & \multicolumn{4}{|c|}{ Previously Treated DME } \\
\hline & \multicolumn{2}{|c|}{$\geq 6$ Months of Follow-up } & \multicolumn{2}{|c|}{$\geq 12$ Months of Follow-up } & \multicolumn{2}{|c|}{$\geq 6$ Months of Follow-up } & \multicolumn{2}{|c|}{$\geq 12$ Months of Follow-up } \\
\hline & $\operatorname{RBZ}(\mathrm{n}=507)$ & $\operatorname{AFL}(n=681)$ & RBZ $(n=326)$ & $\operatorname{AFL}(n=382)$ & RBZ $(n=53)$ & $\operatorname{AFL}(n=223)$ & RBZ $(n=24)$ & $\operatorname{AFL}(n=122)$ \\
\hline \multicolumn{9}{|l|}{ Patient demographics ${ }^{a}$} \\
\hline Age, years, mean (SD) & $62.1(11.2)$ & $63.4(10.8)$ & $62.3(11.5)$ & $64.1(11.2)$ & $60.5 \quad(9.1)$ & $61.4(10.4)$ & $61.2 \quad(9.7)$ & $62.0(10.8)$ \\
\hline Female, n (\%) & $242(47.7)$ & $280(41.1)$ & $156(47.9)$ & $159(41.6)$ & $21(39.6)$ & $105(47.1)$ & $10(41.7)$ & $53(43.4)$ \\
\hline \multicolumn{9}{|l|}{ Index year, n (\%) } \\
\hline 2014 & $177(34.9)$ & $53 \quad(7.8)$ & $145(44.5)$ & $48(12.6)$ & $2 \quad(3.8)$ & $9 \quad(4.0)$ & $1 \quad(4.2)$ & $8 \quad(6.6)$ \\
\hline 2015 & $215(42.4)$ & $381(55.9)$ & $181(55.5)$ & $334(87.4)$ & $33(62.3)$ & $130(58.3)$ & $23(95.8)$ & $114(93.4)$ \\
\hline 2016 & $115(22.7)$ & $247(36.3)$ & $(0)$ & $(0)$ & $18(34.0)$ & $84(37.7)$ & (0) & $(0)$ \\
\hline \multicolumn{9}{|l|}{ Geographic region, $\mathrm{n}(\%)$} \\
\hline Northeast & $131(25.8)$ & $224(32.9)$ & $88 \quad(27.0)$ & $136(35.6)$ & $16(30.2)$ & $62(27.8)$ & $9(37.5)$ & $29(23.8)$ \\
\hline Midwest & $132(26.0)$ & $204(30.0)$ & $83(25.5)$ & $119(31.2)$ & $8(15.1)$ & $48(21.5)$ & $1 \quad(4.2)$ & $31(25.4)$ \\
\hline South & $205(40.4)$ & $211(31.0)$ & $128(39.3)$ & $102(26.7)$ & $22(41.5)$ & $87(39.0)$ & $10(41.7)$ & $49(40.2)$ \\
\hline West & $39 \quad(7.7)$ & $41 \quad(6.0)$ & $27 \quad(8.3)$ & $24 \quad(6.3)$ & $6(11.3)$ & $26(11.7)$ & $4(16.7)$ & $13(10.7)$ \\
\hline Unknown & $(0)$ & $1 \quad(0.1)$ & $(0)$ & $1 \quad(0.3)$ & $1 \quad(1.9)$ & $(0)$ & $(0)$ & $(0)$ \\
\hline \multicolumn{9}{|l|}{ Population density, $\mathrm{n}(\%)$} \\
\hline Urban & $441 \quad(87.0)$ & $584(85.8)$ & $284(87.1)$ & $332(86.9)$ & $48(90.6)$ & $197(88.3)$ & $22(91.7)$ & $112(91.8)$ \\
\hline Rural & $66(13.0)$ & $96(14.1)$ & $42(12.9)$ & $49(12.8)$ & $4 \quad(7.5)$ & $26(11.7)$ & $2 \quad(8.3)$ & $10 \quad(8.2)$ \\
\hline Unknown & $(0)$ & $1 \quad(0.1)$ & $(0)$ & $1 \quad(0.3)$ & $1 \quad(1.9)$ & $(0)$ & (0) & (0) \\
\hline \multicolumn{9}{|l|}{ Health plan type, n (\%) } \\
\hline FFS & $85(16.8)$ & $135(19.8)$ & $59(18.1)$ & $80(20.9)$ & $3 \quad(5.7)$ & $35(15.7)$ & $(0)$ & $26(21.3)$ \\
\hline $\mathrm{HMO}$ & $33 \quad(6.5)$ & $52 \quad(7.6)$ & $19 \quad(5.8)$ & $30 \quad(7.9)$ & $5 \quad(9.4)$ & $19 \quad(8.5)$ & $(0)$ & $8 \quad(6.6)$ \\
\hline POS & $39 \quad(7.7)$ & $37 \quad(5.4)$ & $25 \quad(7.7)$ & $18 \quad(4.7)$ & $2 \quad(3.8)$ & $17 \quad(7.6)$ & $1 \quad(4.2)$ & $6 \quad(4.9)$ \\
\hline $\mathrm{PPO}$ & $288(56.8)$ & $388(57.0)$ & $180(55.2)$ & $217(56.8)$ & $38(71.7)$ & $127(57.0)$ & $19(79.2)$ & $68(55.7)$ \\
\hline Other & $62(12.2)$ & $69(10.1)$ & $43(13.2)$ & $37 \quad(9.7)$ & $5 \quad(9.4)$ & $25(11.2)$ & $4(16.7)$ & $14(11.5)$ \\
\hline \multicolumn{9}{|l|}{ Primary payer type, $\mathrm{n}(\%)$} \\
\hline Commercial & $303(59.8)$ & $379(55.7)$ & $190(58.3)$ & $199(52.1)$ & $40(75.5)$ & $156(70.0)$ & $17(70.8)$ & $80(65.6)$ \\
\hline Medicare & $204(40.2)$ & $302(44.3)$ & $136(41.7)$ & $183(47.9)$ & $13(24.5)$ & $67(30.0)$ & $7(29.2)$ & $42(34.4)$ \\
\hline $\begin{array}{l}\text { Median household } \\
\text { income, USD, mean (SD) }\end{array}$ & $\begin{array}{l}48,273 \\
(17,691) \\
\end{array}$ & $\begin{array}{c}47,763 \\
(15,088) \\
\end{array}$ & $\begin{array}{c}48,621 \\
(17,688)\end{array}$ & $\begin{array}{c}47,887 \\
(14,973) \\
\end{array}$ & $\begin{array}{c}46,382 \\
(13,827)\end{array}$ & $\begin{array}{c}47,652 \\
(17,013) \\
\end{array}$ & $\begin{array}{c}47,382 \\
(14,664) \\
\end{array}$ & $\begin{array}{c}48,242 \\
(16,592)\end{array}$ \\
\hline \multicolumn{9}{|l|}{ Clinical measurements ${ }^{b}$} \\
\hline \multicolumn{9}{|c|}{ Nonophthalmic comorbidities and medications, $\mathrm{n}(\%)$} \\
\hline Nonmelanoma cancer & $32 \quad(6.3)$ & $36 \quad(5.3)$ & $19 \quad(5.8)$ & $21 \quad(5.5)$ & $3 \quad(5.7)$ & $17 \quad(7.6)$ & $1 \quad(4.2)$ & $13(10.7)$ \\
\hline Dyslipidemia & $419(82.6)$ & $551(80.9)$ & $264(81.0)$ & $300(78.5)$ & $46(86.8)$ & $178(79.8)$ & $20(83.3)$ & $99(81.1)$ \\
\hline NSAIDs & $81(16.0)$ & $86(12.6)$ & $53(16.3)$ & $48(12.6)$ & $8(15.1)$ & $24(10.8)$ & $5(20.8)$ & $12(9.8)$ \\
\hline Glucocorticoids & $74(14.6)$ & $100(14.7)$ & $49(15.0)$ & $66(17.3)$ & $6(11.3)$ & $26(11.7)$ & $2(8.3)$ & $17(13.9)$ \\
\hline Diabetes (type 1 or 2 ) & $500(98.6)$ & $676(99.3)$ & $323(99.1)$ & $378(99.0)$ & $52(98.1)$ & $223(100)$ & $23(95.8)$ & $122(100)$ \\
\hline \multicolumn{9}{|c|}{ Ophthalmic comorbidities and treatments, n (\%) } \\
\hline Cataracts & $202(39.8)$ & $291(42.7)$ & $134(41.1)$ & $156(40.8)$ & $25(47.2)$ & $97(43.5)$ & $13(54.2)$ & $47(38.5)$ \\
\hline Glaucoma & $90(17.8)$ & $161(23.6)$ & $70(21.5)$ & $91(23.8)$ & $10(18.9)$ & $40(17.9)$ & $3(12.5)$ & $21(17.2)$ \\
\hline Cataract surgery & $61(12.0)$ & $85(12.5)$ & $43(13.2)$ & $53(13.9)$ & $8(15.1)$ & $24(10.8)$ & $5(20.8)$ & $13(10.7)$ \\
\hline $\begin{array}{l}\text { Intravitreal steroid } \\
\text { injection }\end{array}$ & $10 \quad(2.0)$ & $20 \quad(2.9)$ & $8 \quad(2.5)$ & $13(3.4)$ & $3 \quad(5.7)$ & $12 \quad(5.4)$ & $1 \quad(4.2)$ & $7 \quad(5.7)$ \\
\hline
\end{tabular}

whereas 24 ranibizumab-treated and 122 aflibercept-treated patients were included in 12-month analyses. Across all DME subgroups, baseline demographics and clinical measurements were similar between aflibercept- and ranibizumab-treated patients; however, due to differences in FDA approval dates for aflibercept and ranibizumab in DME, imbalances in index year among treatment-naive patients were observed (Table 2).

\section{Frequency and Cost of Anti-VEGF Injections for nAMD}

Adjusted mean injection frequencies and costs are presented in Figure 1. Among treatment-naive patients with nAMD, there was no significant difference in mean injection frequency between ranibizumab- and aflibercept-treated patients over 12 months (5.62 vs. $5.54 ; P=0.52$ ) or 24 months (7.86 vs. 8.37; $P=0.16$ ). Similarly, mean costs of ranibizumab and aflibercept 
Real-World Injection Frequency and Cost of Ranibizumab and Aflibercept for the Treatment

of Neovascular Age-Related Macular Degeneration and Diabetic Macular Edema

\begin{tabular}{|c|c|c|c|c|c|c|c|c|}
\hline \multirow[b]{3}{*}{ Characteristic } & \multicolumn{4}{|c|}{ Treatment-Naive DME } & \multicolumn{4}{|c|}{ Previously Treated DME } \\
\hline & \multicolumn{2}{|c|}{$\geq 6$ Months of Follow-up } & \multicolumn{2}{|c|}{$\geq 12$ Months of Follow-up } & \multicolumn{2}{|c|}{$\geq 6$ Months of Follow-up } & \multicolumn{2}{|c|}{$\geq 12$ Months of Follow-up } \\
\hline & $\operatorname{RBZ}(\mathrm{n}=507)$ & $\operatorname{AFL}(n=681)$ & RBZ $(n=326)$ & $\operatorname{AFL}(n=382)$ & $\operatorname{RBZ}(\mathrm{n}=53)$ & AFL $(n=223)$ & $\mathrm{RBZ}(\mathrm{n}=24)$ & $\operatorname{AFL}(n=122)$ \\
\hline \multicolumn{9}{|c|}{ Health status indices, mean (SD) } \\
\hline $\begin{array}{l}\text { Deyo Charlson } \\
\text { Comorbidity Index }\end{array}$ & $4.0 \quad(2.0)$ & $4.0 \quad(1.8)$ & $4.1 \quad(2.0)$ & $4.1 \quad(1.8)$ & $4.0 \quad(1.8)$ & $3.9 \quad(1.7)$ & $3.3 \quad(1.6)$ & $4.1 \quad(2.0)$ \\
\hline $\begin{array}{l}\text { Unique ICD-9/10-CM } \\
\text { diagnoses }\end{array}$ & $20.7(13.5)$ & $20.9(14.0)$ & $19.6(12.4)$ & $18.8(11.9)$ & $23.6(16.0)$ & $18.9(11.1)$ & $20.3(13.1)$ & $18.7(11.1)$ \\
\hline $\begin{array}{l}\text { Unique National Drug } \\
\text { Code numbers }\end{array}$ & $17.4 \quad(9.5)$ & $16.0 \quad(9.6)$ & $17.4 \quad(9.4)$ & $16.0 \quad(9.3)$ & $19.7 \quad(9.4)$ & $15.4 \quad(8.8)$ & $19.3 \quad(9.7)$ & $16.1 \quad(9.8)$ \\
\hline $\begin{array}{l}\text { Total health care } \\
\text { expenditure, USD }\end{array}$ & $\begin{array}{l}34,596 \\
(69,816) \\
\end{array}$ & $\begin{array}{c}37,110 \\
(76,856)\end{array}$ & $\begin{array}{l}35,226 \\
(76,991)\end{array}$ & $\begin{array}{l}38,724 \\
(75,452)\end{array}$ & $\begin{array}{c}49,671 \\
(83,217)\end{array}$ & $\begin{array}{c}41,184 \\
(95,272) \\
\end{array}$ & $\begin{array}{c}34,169 \\
(31,497)\end{array}$ & $\begin{array}{l}37,680 \\
(61,027)\end{array}$ \\
\hline $\begin{array}{l}\text { Median total health } \\
\text { care expenditure, USD }\end{array}$ & 16,155 & 16,696 & 15,726 & 16,539 & 23,331 & 18,363 & 24,837 & 18,619 \\
\hline \multicolumn{9}{|c|}{ Unadjusted frequency and cost of anti-VEGF injections $\mathrm{s}^{\mathrm{c}}$} \\
\hline $\begin{array}{l}\text { Injection frequency, } \\
\text { mean (SD) }\end{array}$ & $2.60(1.62)$ & $2.92(1.61)$ & $3.15(2.55)$ & $3.80(2.58)$ & $2.94(1.74)$ & $3.09(1.65)$ & $3.38(2.55)$ & $4.29(2.89)$ \\
\hline$P$ value & \multicolumn{2}{|c|}{$<0.001$} & \multicolumn{2}{|c|}{$<0.001$} & \multicolumn{2}{|c|}{0.56} & \multicolumn{2}{|c|}{0.15} \\
\hline $\begin{array}{l}\text { Injection cost, USD, } \\
\text { mean (SD) }\end{array}$ & $\begin{array}{c}3,376 \\
(2,526)\end{array}$ & $\begin{array}{c}5,929 \\
(3,966)\end{array}$ & $\begin{array}{c}4,145 \\
(3,830)\end{array}$ & $\begin{array}{c}7,627 \\
(5,496)\end{array}$ & $\begin{array}{c}3,711 \\
(2,282)\end{array}$ & $\begin{array}{c}6,815 \\
(5,001)\end{array}$ & $\begin{array}{c}4,011 \\
(3,138)\end{array}$ & $\begin{array}{c}9,424 \\
(8,259)\end{array}$ \\
\hline$P$ value & \multicolumn{2}{|c|}{$<0.001$} & \multicolumn{2}{|c|}{$<0.001$} & \multicolumn{2}{|c|}{$<0.001$} & \multicolumn{2}{|c|}{0.002} \\
\hline \multicolumn{9}{|c|}{$\begin{array}{l}\text { aPatient demographics were measured at the index date for each anti-VEGF treatment exposure. } \\
{ }^{2} \text { Clinical measurements were measured during the } 12 \text {-month baseline period for each anti-VEGF treatment exposure. } \\
\text { cUnadjusted injection frequencies and costs were measured over the specified length of follow-up (6 and } 12 \text { months). In patients with }>6 \text { and }>12 \text { months of follow-up, } \\
\text { data were censored after the first } 6 \text { and } 12 \text { months, respectively. } \\
\text { AFL=aflibercept; DME = diabetic macular edema; FFS = fee-for-service; HMO=health maintenance organization; ICD-9/10-CM=International Classification of } \\
\text { Diseases, Ninth/Tenth Revision, Clinical Modification; NSAID=nonsteroidal anti-inflammatory drug; POS=point-of-service; PPO=preferred provider organization; } \\
\text { RBZ=ranibizumab; SD=standard deviation; USD=U.S. dollars; VEGF=vascular endothelial growth factor. }\end{array}$} \\
\hline
\end{tabular}

did not differ significantly at 12 months ( $\$ 11,351$ vs. $\$ 10,702$; $P=0.06)$ or 24 months ( $\$ 16,286$ vs. $\$ 16,666 ; P=0.69)$. In previously treated patients with nAMD, there was no significant difference between ranibizumab and aflibercept for mean number of injections (5.95 vs. $6.09 ; P=0.56$ ) or treatment costs $(\$ 11,512$ vs. $\$ 12,050 ; P=0.44)$ at 12 months. In 24 -month analyses, mean injection frequency was significantly lower for ranibizumab (7.98 vs. 9.63; $P=0.03)$; however, differences in ranibizumab and aflibercept treatment costs were not statistically significant ( $\$ 16,303$ vs. $\$ 19,361 ; P=0.13)$.

\section{Frequency and Cost of Anti-VEGF Injections for DME}

As shown in Figure 2, adjusted mean injection frequency among treatment-naive patients with DME was significantly lower with ranibizumab versus aflibercept over 6 months (2.60 vs. 2.92; $P<0.001)$ and 12 months (3.33 vs. 3.87; $P<0.001)$. Similarly, adjusted mean injection costs were significantly lower in ranibizumab-treated patients at 6 months $(\$ 3,379$ vs. $\$ 5,925 ; P<0.0001)$ and 12 months $(\$ 4,136$ vs. $\$ 7,656 ; P<0.0001$ ). In previously treated patients with DME, there was no significant difference between the mean number of ranibizumab and aflibercept injections received over 6 months
(3.01 vs. $3.08 ; P=0.80$ ) or 12 months (3.81 vs. $4.19 ; P=0.59$ ). Despite this, mean injection costs were significantly lower for ranibizumab versus aflibercept over 6 months $(\$ 3,834$ vs. $\$ 6,775 ; P=0.0001$ ) and 12 months ( $\$ 4,606$ vs. $\$ 9,190 ; P=0.02$ ).

\section{Distribution of Anti-VEGF Injections for $\mathrm{AAMD}$ and DME}

When the number of anti-VEGF injections received by treatment-naive patients with nAMD and DME were grouped according to FDA-indicated injection schedules, treatment patterns were similar between those who received ranibizumab versus aflibercept (Figure 3). The majority of ranibizumab-and aflibercept-treated patients with nAMD received $\geq 3$ injections over 12 months of follow-up ( $79.0 \%$ vs. $81.6 \%$, respectively), whereas $41.4 \%$ versus $41.0 \%$ received $\geq 7$ injections, $21.0 \%$ versus $18.4 \%$ received $1-2$ injections, and only $6.6 \%$ versus $4.6 \%$ received $\geq 11$ (i.e., $\mathrm{q} 4 \mathrm{w}$ ) injections. In ranibizumaband aflibercept-treated patients with nAMD and 24 months of follow-up, $78.9 \%$ versus $84.3 \%$ received $\geq 3$ injections, $23.1 \%$ versus $25.5 \%$ received $\geq 13$ injections, $21.2 \%$ versus $15.7 \%$ received $1-2$ injections, and $0.9 \%$ in each group received $\geq 23$ (i.e., q4w) injections (data not shown). 

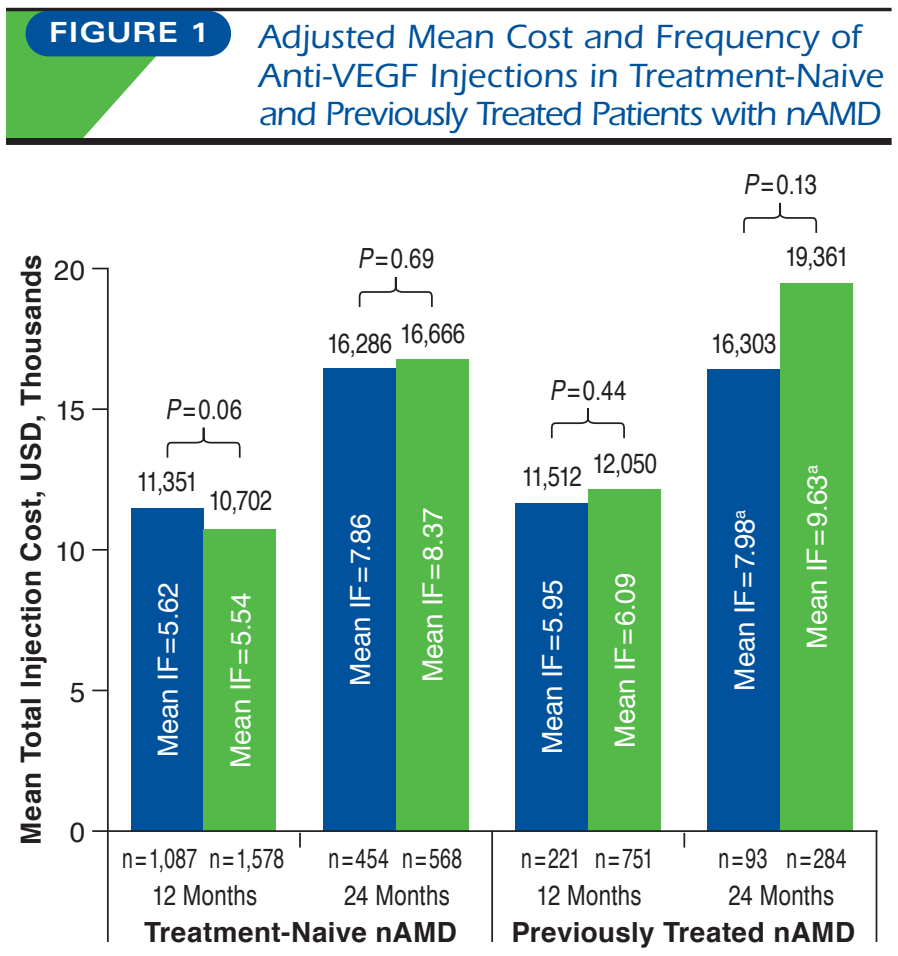

Ranibizumab Aflibercept

Note: Poisson quasi-likelihood multivariable regression was used to model injection frequencies; multivariable generalized linear models with a log link and gamma error distribution were used to model total injection costs over the specified length of follow-up. Analyses were adjusted for patient demographics, baseline clinical characteristics, indices of general health status, baseline treatments, and other covariates.

asignificant difference in mean IF between ranibizumab-and aflibercept-treated patients at 24 months $(P=0.03)$.

IF = injection frequency; $n A M D=$ neovascular age-related macular degeneration; $U S D=U . S$. dollars; VEGF = vascular endothelial growth factor.

More than 90\% of treatment-naive patients with DME received $<8$ ranibizumab or aflibercept injections in the year following treatment initiation (Figure 3). More specifically, the majority of ranibizumab- and aflibercept-treated patients with DME received 1-4 injections over 12 months of follow-up (77.0\% vs. $66.5 \%$, respectively), whereas $23.0 \%$ versus $33.5 \%$ received $\geq 5$ injections, $8.9 \%$ versus $9.4 \%$ received $\geq 8$ injections, and only $2.1 \%$ in each group received $\geq 11$ (i.e., q4w) injections.

\section{Discussion}

This real-world retrospective analysis provided valuable insight regarding treatment frequency and health care resource utilization associated with ranibizumab and aflibercept therapy among patients with nAMD and DME. Our findings suggest that ranibizumab treatment for DME may be cost saving compared with aflibercept and that anti-VEGF treatment
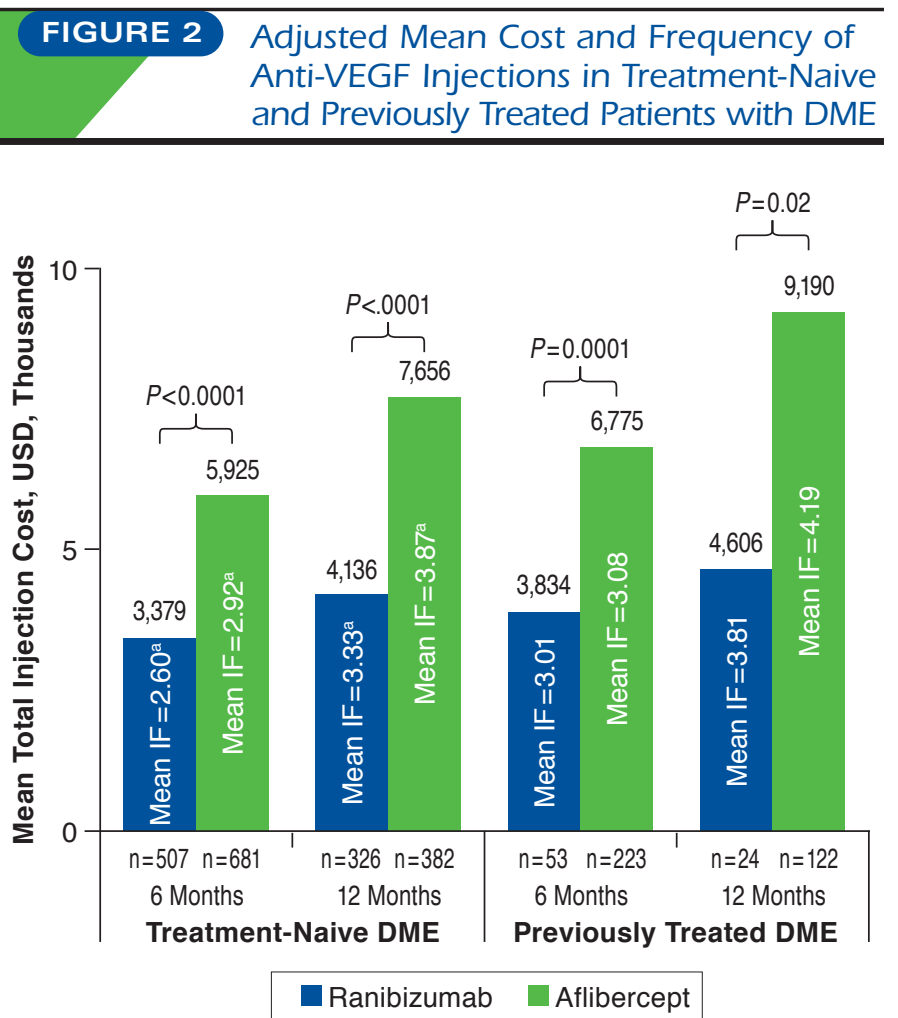

Note: Poisson quasi-likelihood multivariable regression was used to model injection frequencies; multivariable generalized linear models with a log link and gamma error distribution were used to model total injection costs over the specified length of follow-up. Analyses were adjusted for patient demographics, baseline clinical characteristics, indices of general health status, baseline treatments, and other covariates.

asignificant difference in mean IF between ranibizumab-and aflibercept-treated patients at 6 and 12 months (both $P<0.001$ ).

$D M E=$ diabetic macular edema; IF = injection frequency; USD =U.S. dollars; $V E G F=$ vascular endothelial growth factor.

patterns in clinical practice are not reflective of FDA-indicated dosing schedules.

\section{Interpretation of Results}

Of the nAMD cohorts evaluated, estimated costs and injection frequencies were comparable between ranibizumab and aflibercept in treatment-naive patients over 12 and 24 months, consistent with previous findings. ${ }^{24,25}$ Comparable results were also observed in previously treated nAMD cohorts over 12 and 24 months, with the exception that injection frequency at 24 months was lower for patients receiving ranibizumab versus aflibercept. Relative to other nAMD subgroups, 24-month analyses of the previously treated cohort were based on a small number of patients (particularly those receiving ranibizumab; $\mathrm{n}=93$ ) and should therefore be interpreted with caution. 


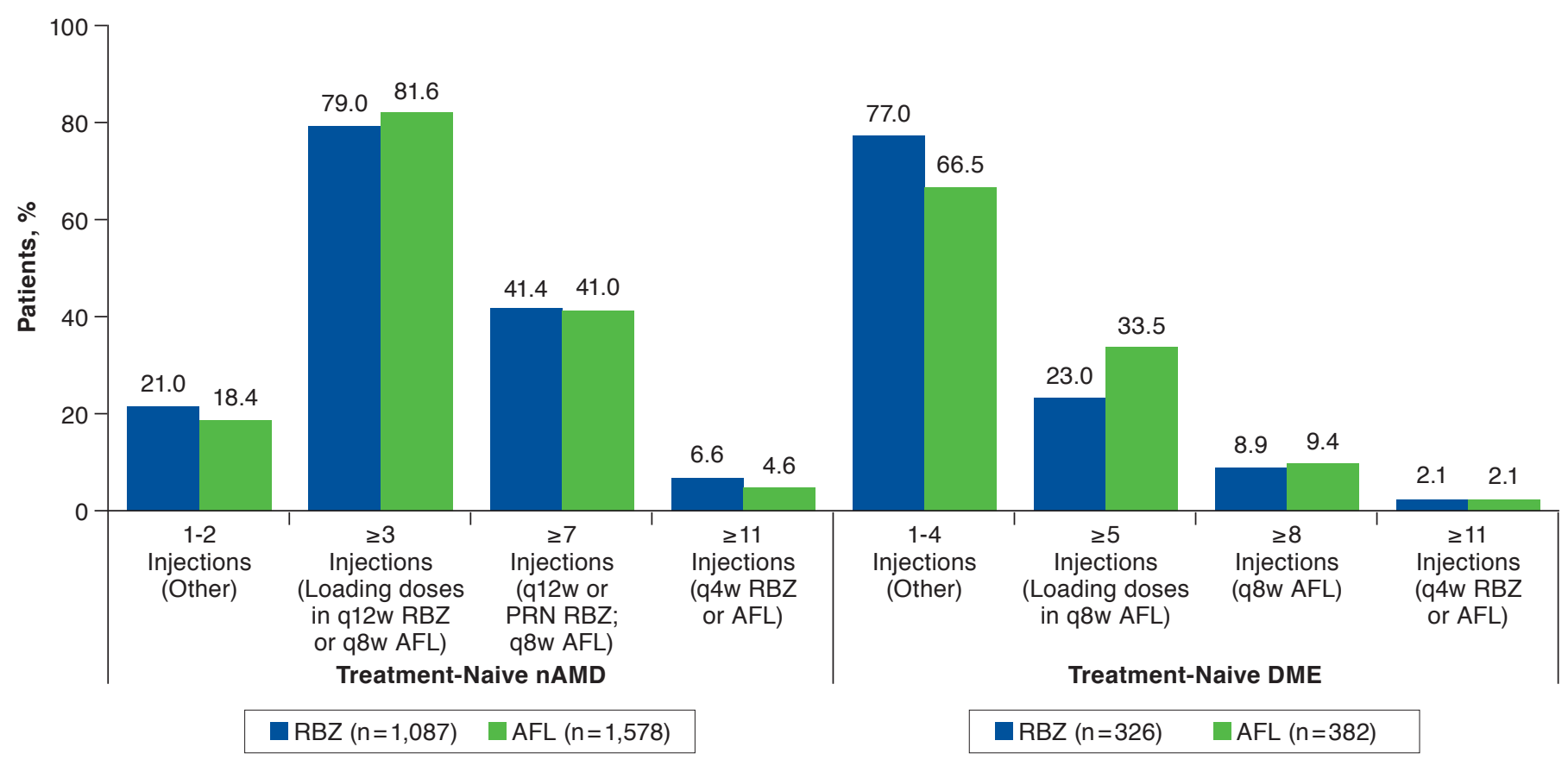

Note: Injection thresholds used to define dosing categories included an allowable margin of 1 injection, to account for strict cutoffs to follow-up in this study (e.g., q4w dosing over 12 months was defined as $\geq 11$ injections, rather than $\geq 12$ injections).

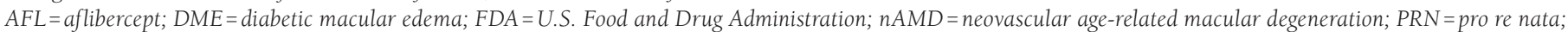
$q 4 w=$ monthly; $q 8 w=$ every 2 months; $q 12 w=$ every 3 months; RBZ= ranibizumab; VEGF=vascular endothelial growth factor.

Of the DME cohorts evaluated, initiation of ranibizumab in treatment-naive and previously treated patients was associated with lower treatment costs than aflibercept over 6 and 12 months. Cost savings associated with ranibizumab for DME were likely driven by the lower cost of ranibizumab $0.3 \mathrm{mg}$ (WAC \$1,170) compared with aflibercept $2.0 \mathrm{mg}$ (WAC \$1,850). Significant differences in mean injection frequency may account for additional cost savings among treatment-naive patients with DME; however, mean differences were $<1$ injection between those receiving ranibizumab versus aflibercept. Our 12-month analyses found that the mean cost of ranibizumab therapy was 46\%-50\% lower than aflibercept in treatment-naive and previously treated patients with DME, resulting in estimated per-patient cost savings of $\$ 3,500$ $\$ 4,500$ over 1 year of treatment.

Although we found statistically significant differences in mean injection frequency between ranibizumab- and aflibercept-treated patients in the treatment-naive DME cohort, it is unclear whether such differences $(<1$ injection over 12 months) may be considered meaningful in clinical practice. For example, observed differences in injection frequency may translate to differences in real-world vision outcomes achieved by ranibizumab- and aflibercept-treated patients; however, we were unable to explore this relationship due to a paucity of visual acuity data within insurance claims databases.

\section{Real-World Dosing Versus Labeled Injection Schedules}

Relative to ranibizumab, current drug labels suggest that fewer aflibercept injections may be required for the ongoing treatment of nAMD and DME. ${ }^{13,14}$ However, our analyses support the notion that real-world anti-VEGF treatment patterns, on average, do not reflect FDA-approved dosing schedules. These findings are consistent with previous analyses of real-world anti-VEGF treatment patterns, which have similarly reported average 12-month injection frequencies of 4.3-5.8 and 2.2-4.4 among patients with nAMD and DME, respectively. ${ }^{24,25,33-38}$ Our results also agree with American Society of Retina Specialists Preferences and Trends Surveys, which suggest that most retina specialists prefer to administer ongoing treatment according to anatomic findings observed on follow-up optical coherence tomography (e.g., subfoveal retinal thickness and central subfield thickness), rather than FDA labeling. ${ }^{21,22}$ However, these findings are not intended to suggest that real-world injection frequencies reflect patient need or response to therapy. 


\section{Real-World Injection Frequency and Cost of Ranibizumab and Aflibercept for the Treatment of Neovascular Age-Related Macular Degeneration and Diabetic Macular Edema}

Rather, the number of injections administered in clinical practice likely depends on multiple factors, including but not limited to patient adherence, treatment response, and patient/ clinician preference. In any case, our observation that a significant proportion of treatment-naive patients with nAMD and DME received fewer ranibizumab and aflibercept injections than any FDA-indicated schedule is a potential cause for concern and may result in suboptimal vision outcomes in clinical practice.

Research in nAMD suggests that although acceptable vision gains are achievable with less frequent anti-VEGF treatment, many patients require more frequent injections to achieve optimal outcomes. The PIER trial reported rapid vision improvements in patients who received 3 monthly loading doses of ranibizumab; however, this benefit was lost with $\mathrm{q} 12 \mathrm{w}$ dosing between months 3 and $12 .{ }^{39}$ One-year vision outcomes with $\mathrm{q} 12 \mathrm{w}$ ranibizumab were more favorable in EXCITE but remained inferior to $\mathrm{q} 4 \mathrm{w}$ dosing. ${ }^{40}$ In the Comparison of Agerelated Macular Degeneration Treatments Trials and HARBOR, mean vision gains among ranibizumab- and bevacizumabtreated patients were greater in those who received $q 4 \mathrm{w}$ versus PRN treatment, ${ }^{29,41}$ whereas in VIEW 1 and VIEW 2, small reductions in visual improvement were observed in patients who switched from $\mathrm{q} 4 \mathrm{w}$ or $\mathrm{q} 8 \mathrm{w}$ aflibercept during year 1 , to at least $\mathrm{q} 12 \mathrm{w}$ dosing during year $2 .^{18}$

\section{Limitations}

Insurance claims data provide valuable insight into real-world treatment patterns, health care resource utilization, and patient outcomes; however, there are several limitations associated with their use in health economic research. Our analyses may be subject to errors within the MarketScan Research Databases, particularly with respect to treatment codes and costs.

Aflibercept entered the market before receiving productspecific HCPCS codes; therefore, we identified probable aflibercept therapy during this period using nonspecific HCPCS codes and concurrent Current Procedural Terminology codes indicating intravitreal injection. More generally, we excluded patients with evidence of bilateral anti-VEGF treatment because the inconsistent use of modifiers within claims data do not allow for identification of the treated eye.

With regard to costs, our sample reflects only paid claims and excluded situations in which anti-VEGF therapy was administered outside an insurance plan. Although cost analyses did not consider treatment-related adverse events, previous trials in $\mathrm{nAMD}$ and DME have shown that the incidence of ocular and systemic adverse events are overall low and similar between ranibizumab- and aflibercept-treated patients. ${ }^{17-19}$ Similarly, given that ranibizumab and aflibercept injection frequencies were generally comparable across patient subgroups in this study, we believe that the exclusion of administration costs from our analyses has not significantly impacted our results and overall conclusions.

Caution is required when extrapolating our results to describe anti-VEGF treatment patterns across the wider U.S. population. MarketScan Research Databases do not include all payers, uninsured patients, or those insured via Medicaid or Medicare fee-for-service, and its patient sample is not proportionally weighted by U.S. region. We did not examine treatment patterns and costs associated with off-label bevacizumab therapy, and we were unable to account for potential changes to nAMD and DME management after December 31, 2016.

Moreover, the FDA approval date for aflibercept in DME (July 29, 2014) precluded a 24-month analysis in this population due to an insufficient number of patients with adequate follow-up. Observed differences in index date between treatment-naive DME cohorts reflected differences in FDA approval dates between aflibercept and ranibizumab (August 10, 2012); thus, our 2014 sample was weighted toward ranibizumab treatment, whereas our 2015-2016 sample was weighted toward aflibercept treatment. Future studies with extended follow-up periods will further improve comparisons among patients with DME and will allow characterization of longer-term anti-VEGF treatment patterns.

\section{Strengths}

Despite its limitations, the MarketScan Research Databases collectively represent one of the longest-running and largest repositories of high-quality health care data in the United States. ${ }^{26}$ To capture the most recent anti-VEGF treatment patterns and avoid potential biases, our nAMD cohort excluded patients who initiated therapy before January 1, 2014, and our DME cohort excluded patients treated with ranibizumab before FDA approval of aflibercept in this indication.

A study end date of December 31, 2016, ensured that $\geq 95 \%$ of claims during follow-up were adjudicated, whereas the inclusion of treatment-naive and previously treated subgroups provided the opportunity to compare anti-VEGF treatment patterns between these patient populations.

Our analyses were adjusted to account for confounding patient demographics, baseline clinical characteristics, and other covariates; however, we were unable to control for potential confounders not captured within insurance claims databases (e.g., anatomic and visual acuity data).

Finally, costs extracted from claims data are based on reimbursement expenses, which are more reflective of actual costs than list or other drug price estimates. To ensure objectivity, no paid claims were removed from our analyses (including claims ranging from $\$ 0$ to $>\$ 30,000$ ). 


\section{Real-World Injection Frequency and Cost of Ranibizumab and Aflibercept for the Treatment of Neovascular Age-Related Macular Degeneration and Diabetic Macular Edema}

\section{Conclusions}

Although the real-world frequency of ranibizumab and aflibercept injections were generally comparable among patients with nAMD and DME in this study, ranibizumab therapy was associated with estimated per-patient cost savings of $\$ 3,500-\$ 4,500$ over 1 year of treatment in patients with DME. However, a significant proportion of patients received fewer than the recommended number of ranibizumab or aflibercept injections for nAMD and DME, suggesting potential undertreatment that may lead to suboptimal vision outcomes. Given that labeled dosing schedules are informed by landmark clinical trials, our data highlight a need to identify barriers to optimal treatment in routine clinical practice, and to develop new strategies that improve anti-VEGF utilization and associated patient outcomes.

\section{Authors}

SZILÁRD KISS, MD, Weill Cornell Medical College, New York, New York. ELISABETTA MALANGONE-MONACO, MS; KATHLEEN WILSON, MPH; HELEN VARKER, BS; DIANA STETSOVSKY, MS; and DAVID SMITH, PhD, IBM Watson Health, Cambridge, Massachusetts. VINCENT GARMO, MHS, Genentech, South San Francisco, California.

AUTHOR CORRESPONDENCE: Szilárd Kiss, MD, Weill Cornell Medical College, New York-Presbyterian Hospital, 1305 York Ave., New York, NY 10021. Tel.: 646.962.2217; E-mail: szk7001@med.cornell.edu.

\section{REFERENCES}

1. Congdon N, O'Colmain B, Klaver CC, et al; Eye Diseases Prevalence Research Group. Causes and prevalence of visual impairment among adults in the United States. Arch Ophthalmol. 2004;122(4):477-85.

2. Zhang X, Saaddine JB, Chou C-F, et al. Prevalence of diabetic retinopathy in the United States, 2005-2008. JAMA. 2010;304(6):649-56.

3. Klein R, Chou C-F, Klein BE, Zhang X, Meuer SM, Saaddine JB.

Prevalence of age-related macular degeneration in the US population. Arch Ophthalmol. 2011;129(1):75-80

4. Zarbin MA. Current concepts in the pathogenesis of age-related macular degeneration. Arch Ophthalmol. 2004;122(4):598-614.

5. Daruich A, Matet A, Moulin A, et al. Mechanisms of macular edema: beyond the surface. Prog Retin Eye Res. 2018;63:20-68.

6. Lopez PF, Sippy BD, Lambert HM, Thach AB, Hinton DR

Transdifferentiated retinal pigment epithelial cells are immunoreactive for vascular endothelial growth factor in surgically excised age-related macular degeneration-related choroidal neovascular membranes. Invest Ophthalmol Vis Sci. 1996;37(5):855-68

7. Kvanta A, Algvere P, Berglin L, Seregard S. Subfoveal fibrovascular membranes in age-related macular degeneration express vascular endothelial growth factor. Invest Ophthalmol Vis Sci. 1996;37(9):1929-34.

8. Kliffen M, Sharma HS, Mooy CM, Kerkvliet S, de Jong PT. Increased expression of angiogenic growth factors in age-related maculopathy. $\mathrm{Br} \mathrm{J}$ Ophthalmol. 1997;81(2):154-62

9. Funatsu H, Yamashita H, Ikeda T, Nakanishi Y, Kitano S, Hori S. Angiotensin II and vascular endothelial growth factor in the vitreous fluid of patients with diabetic macular edema and other retinal disorders. Am J Ophthalmol. 2002;133(4):537-43

10. Funatsu H, Noma H, Mimura T, Eguchi S, Hori S. Association of vitreous inflammatory factors with diabetic macular edema. Ophthalmology. 2009;116(1):73-79.

11. Flaxel CJ, Adelman RA, Bailey ST, et al. Age-Related Macular Degeneration Preferred Practice Pattern®. Ophthalmology. 2020;127(1):P1-P65.

12. Flaxel CJ, Adelman RA, Bailey ST, et al. Diabetic Retinopathy Preferred Practice Pattern (B. Ophthalmology. 2020;127(1):P66-P145

13. Lucentis (ranibizumab injection) for intravitreal injection. Genentech. March 2018. Available at: https://www.gene.com/download/pdf/lucentis_ prescribing.pdf. Accessed January 16, 2020.

14. Eylea (aflibercept) injection, for intravitreal use. Regeneron Pharmaceuticals. May 2019. Available at: https://www.regeneron.com/sites/ default/files/EYLEA_FPI.pdf. Accessed January 16, 2020.

15. Macugen (pegaptanib sodium injection) intravitreal injection. Bausch \& Lomb International, July 2016. Available at: http://www.bausch.com/Portals/77//m/BL/United\%20States/Files/Package\%20Inserts/Pharma/macugen-packageinsert.pdf?ver=2017-02-14-083844-843. Accessed January 16, 2020.

16. Avastin (bevacizumab) injection, for intravenous use. Genentech. June 2019. Available at: https://www.gene.com/download/pdf/avastin_prescribing.pdf. Accessed January 16, 2020.

17. Heier JS, Brown DM, Chong V, et al; VIEW 1 and VIEW 2 Study Groups. Intravitreal aflibercept (VEGF Trap-Eye) in wet age-related macular degeneration. Ophthalmology. 2012;119(12):2537-48.

18. Schmidt-Erfurth U, Kaiser PK, Korobelnik J-F, et al. Intravitreal aflibercept injection for neovascular age-related macular degeneration: ninety-sixweek results of the VIEW studies. Ophthalmology. 2014;121(1):193-201.

19. Wells JA, Glassman AR, Ayala AR, et al; Diabetic Retinopathy Clinical Research Network. Aflibercept, bevacizumab, or ranibizumab for diabetic macular edema. N Engl J Med. 2015;372(13):1193-203.

20. Wells JA, Glassman AR, Ayala AR, et al. Aflibercept, bevacizumab, or ranibizumab for diabetic macular edema: two-year results from a comparative effectiveness randomized clinical trial. Ophthalmology. 2016;123(6):1351-59. 


\section{Real-World Injection Frequency and Cost of Ranibizumab and Aflibercept for the Treatment of Neovascular Age-Related Macular Degeneration and Diabetic Macular Edema}

21. Stone TW. American Society of Retina Specialists 2018 Preferences and Trends Survey. 2018. Available at: https://www.asrs.org/asrs-community/patsurvey. Accessed January 28, 2020.

22. Stone TW. American Society of Retina Specialists 2015 Preferences and Trends Survey. 2015. Available at: https://www.asrs.org/asrs-community/patsurvey. Accessed January 28, 2020.

23. Calvo P, Wang Y, Ferreras A, Lam W-C, Devenyi R, Brent MH. Treat and extend versus treat and observe regimens in wet age-related macular degeneration patients treated with ranibizumab: 3-year surveillance period. J Clin Exp Ophthalmol. 2014;5(1):1-5.

24. Johnston SS, Wilson K, Huang A, Smith D, Varker H, Turpcu A. Retrospective analysis of first-line anti-vascular endothelial growth factor treatment patterns in wet age-related macular degeneration. Adv Ther. 2013;30(12):1111-27.

25. Ferreira A, Sagkriotis A, Olson M, Lu J, Makin C, Milnes F. Treatment frequency and dosing interval of ranibizumab and aflibercept for neovascular age-related macular degeneration in routine clinical practice in the USA. PLoS One. 2015;10(7):e0133968.

26. IBM Watson Health. IBM MarketScan Research Databases for life sciences researchers. April 2019. Available at: https://www.ibm.com/downloads/ cas/OWZWJOQO. Accessed January 17, 2020.

27. National Center for Health Statistics. International Classification of Diseases, Ninth Revision, Clinical Modification (ICD-9-CM). November 6, 2015. Available at: https://www.cdc.gov/nchs/icd/icd9cm.htm. Accessed January 17, 2020.

28. National Center for Health Statistics. International Classification of Diseases, Tenth Revision, Clinical Modification (ICD-10-CM). January 22, 2020. Available at: https://www.cdc.gov/nchs/icd/icdl0cm.htm. Accessed January 28, 2020.

29. Busbee BG, Ho AC, Brown DM, et al; HARBOR Study Group. Twelvemonth efficacy and safety of $0.5 \mathrm{mg}$ or $2.0 \mathrm{mg}$ ranibizumab in patients with subfoveal neovascular age-related macular degeneration. Ophthalmology. 2013;120(5):1046-56.

30. Ho AC, Busbee BG, Regillo CD, et al; HARBOR Study Group. Twentyfour-month efficacy and safety of $0.5 \mathrm{mg}$ or $2.0 \mathrm{mg}$ ranibizumab in patients with subfoveal neovascular age-related macular degeneration. Ophthalmology. 2014;121(11):2181-92.
31. Deyo RA, Cherkin DC, Ciol MA. Adapting a clinical comorbidity index for use with ICD-9-CM administrative databases. J Clin Epidemiol. 1992;45(6):613-19.

32. U.S. Food \& Drug Administration. National Drug Code Directory. November 18, 2019. Available at: https://www.fda.gov/Drugs/ InformationOnDrugs/ucml42438.htm. Accessed January 17, 2020.

33. Cohen SY, Mimoun G, Oubraham H, et al; LUMIERE Study Group. Changes in visual acuity in patients with wet age-related macular degeneration treated with intravitreal ranibizumab in daily clinical practice: the LUMIERE study. Retina. 2013;33(3):474-81.

34. Finger RP, Wiedemann P, Blumhagen F, Pohl K, Holz FG. Treatment patterns, visual acuity and quality-of-life outcomes of the WAVE study: a noninterventional study of ranibizumab treatment for neovascular age-related macular degeneration in Germany. Acta Ophthalmol. 2013;91(6):540-46.

35. Holz FG, Tadayoni R, Beatty S, et al. Multi-country real-life experience of anti-vascular endothelial growth factor therapy for wet age-related macular degeneration. Br J Ophthalmol. 2015;99(2):220-26.

36. Kiss S, Liu Y, Brown J, et al. Clinical utilization of anti-vascular endothelial growth-factor agents and patient monitoring in retinal vein occlusion and diabetic macular edema. Clin Ophthalmol. 2014;8:1611-21.

37. Campbell J, Cole AL, Almony A, et al. Real world vision outcomes in DME treated with anti-VEGF injections - an analysis of EMR data from a large health system. Invest Ophthalmol Vis Sci. 2014;55(13):3065.

38. Lai TT, Yang C-M, Yang C-H, Ho T-C, Hsieh Y-T. Treatment outcomes and predicting factors for diabetic macular edema treated with ranibizumab one-year real-life results in Taiwan. J Formos Med Assoc. 2019;118(1):194-202.

39. Regillo CD, Brown DM, Abraham P, et al. Randomized, double-masked, sham-controlled trial of ranibizumab for neovascular age-related macular degeneration: PIER study year 1. Am J Ophthalmol. 2008;145(2):239-48.

40. Schmidt-Erfurth U, Eldem B, Guymer R, et al; EXCITE Study Group. Efficacy and safety of monthly versus quarterly ranibizumab treatment in neovascular age-related macular degeneration: the EXCITE study. Ophthalmology. 2011;118(5):831-39.

41. Martin DF, Maguire MG, Fine SL, et al; Comparison of Age-related Macular Degeneration Treatments Trials (CATT) Research Group. Ranibizumab and bevacizumab for treatment of neovascular age-related macular degeneration: two-year results. Ophthalmology. 2012;119(7):1388-98. 


\section{Real-World Injection Frequency and Cost of Ranibizumab and Aflibercept for the Treatment of Neovascular Age-Related Macular Degeneration and Diabetic Macular Edema}

\section{APPENDIX A Patient Selection for nAMD Subgroups}

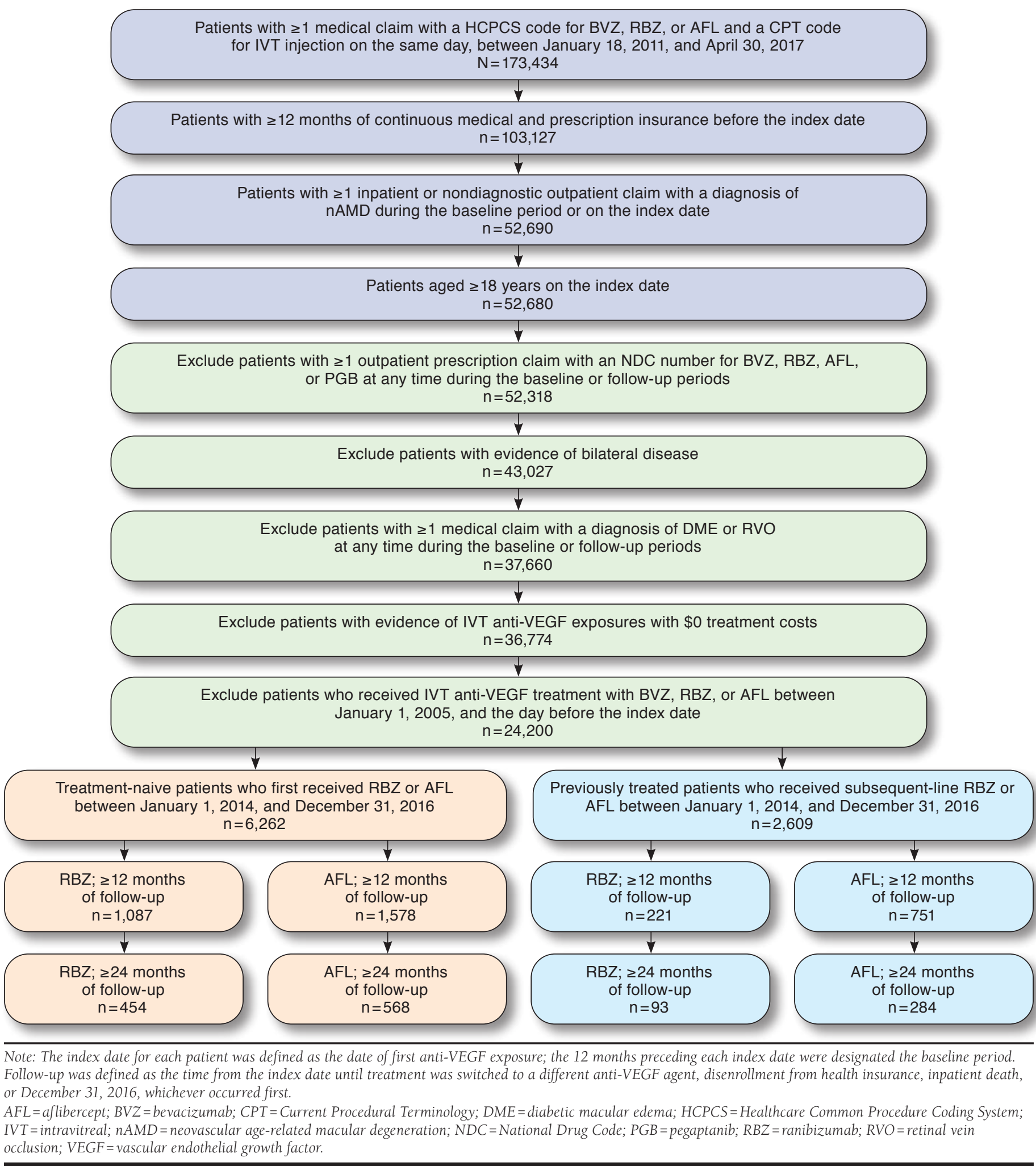




\section{Real-World Injection Frequency and Cost of Ranibizumab and Aflibercept for the Treatment of Neovascular Age-Related Macular Degeneration and Diabetic Macular Edema}

\section{APPENDIX B Patient Selection for DME Subgroups}

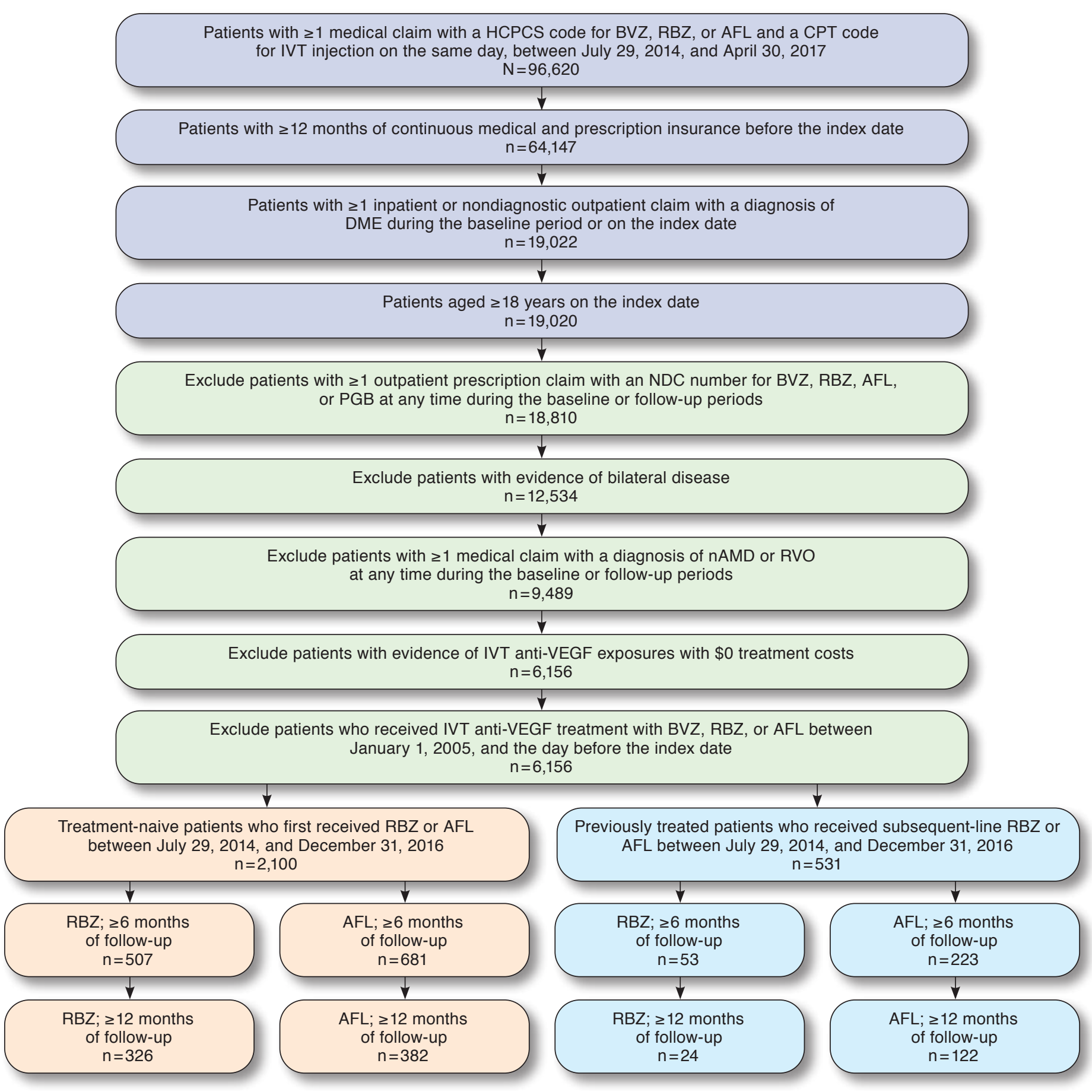

Treatment-naive patients who first received RBZ or AFL between July 29, 2014, and December 31, 2016

$$
n=2,100
$$

Note: The index date for each patient was defined as the date of first anti-VEGF exposure; the 12 months preceding each index date were designated the baseline period. Follow-up was defined as the time from the index date until treatment was switched to a different anti-VEGF agent, disenrollment from health insurance, inpatient death, or December 31, 2016, whichever occurred first.

$A F L=$ aflibercept; $B V Z=$ bevacizumab; $C P T=$ Current Procedural Terminology; DME = diabetic macular edema; HCPCS = Healthcare Common Procedure Coding System; $I V T=$ intravitreal; $n A M D=$ neovascular age-related macular degeneration; $N D C=$ National Drug Code; $P G B=$ pegaptanib; $R B Z=$ ranibizumab; $R V O=$ retinal vein occlusion; VEGF = vascular endothelial growth factor. 


\section{AMCP}
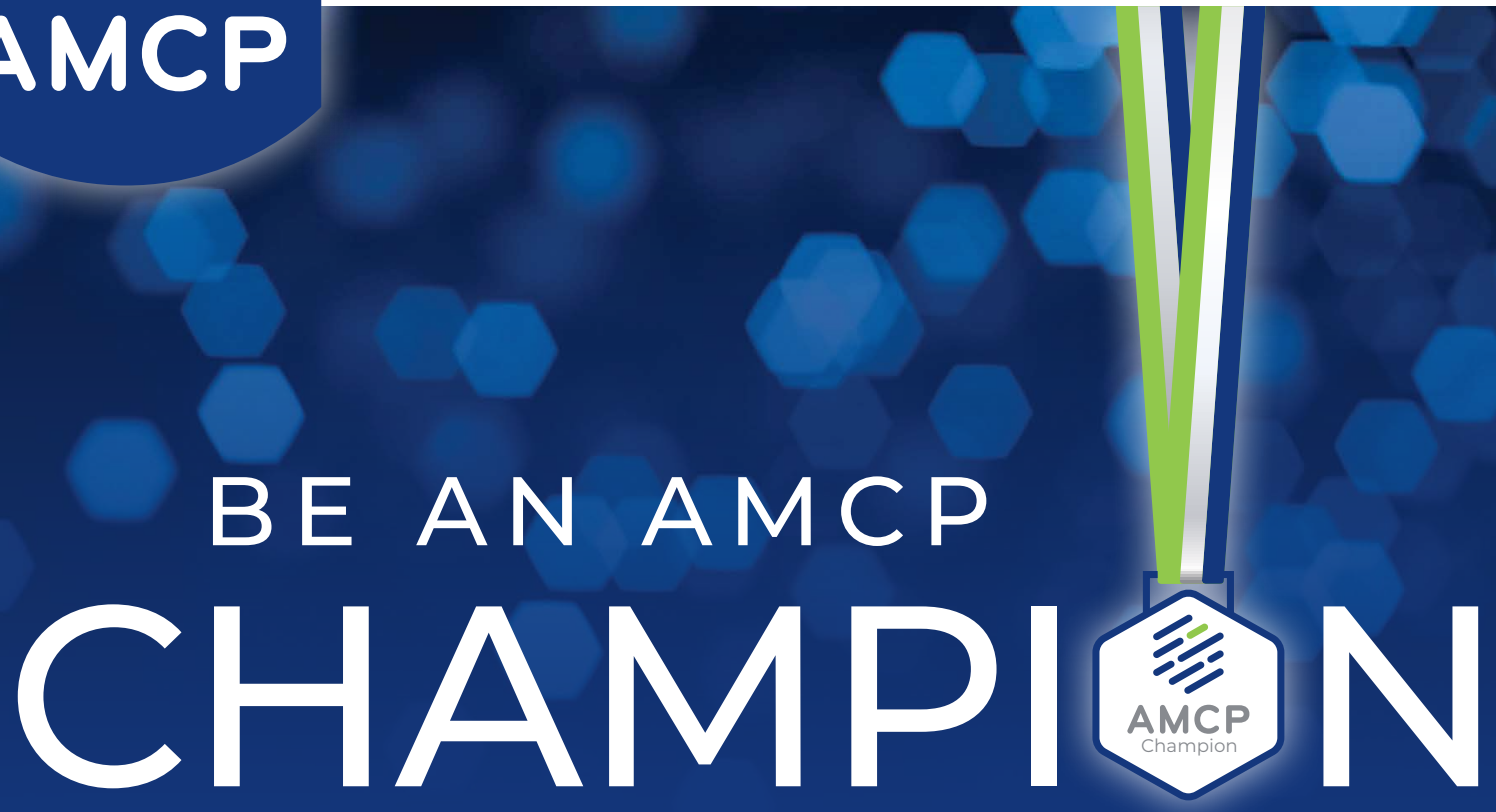

\section{Champion Rewards}

- CHAMPION (1-3 Member Referrals) An AMCP Champion pin

- BRONZE (4-7 Member Referrals) - Above, plus a \$5 Starbucks gift card

- SILVER (8-14 Member Referrals) - Above, plus an AMCP Champion winter scarf

- GOLD (15-24 Member Referrals) - Above, plus an AMCP Champion polo

- PLATINUM (25+ Member Referrals) - Above, plus an AMCP Champion fleece jacket

All levels are recognized on the AMCP website amcp.org/hall-of-fame - and at AMCP meetings.

\section{Recruiting your colleagues is easy.}

Check out these recruitment tools and learn more: www.amcp.org/champion

\section{Champion noun}

cham.pi.on

An AMCP member who actively promotes how their membership in the organization has positively impacted their career and the populations they serve. Helps strengthen managed care pharmacy by inviting their colleagues to join $A M C P$.

As a Platinum Level Champion, I challenge all of our members to strive to achieve this milestone. Many of those I've recruited thanked me for encouraging them and shared it was one of their best decisions!

Rusty Hailey, PharmD, DPh, MBA, FAMCP, AMCP Past President Vice President, Medicaid Pharmacy Services Change HealthCare 